incarcerated small bowel hernia sac has been reported [10]. We describe the first reported case of a plastic biliary stent causing perforation inside a parastomal hernia.

Key words: Stent - Biliary - Perforation - Hernia Correspondence to: J. M. Levey

\section{The hourglass appearance of the neurofibroma on rectal ultrasound}

\section{A case report}

M. Kassai, ${ }^{1}$ L. Illényi,${ }^{1}$ Ö. P. Horváth, ${ }^{1}$ A. Macdonald ${ }^{2}$

${ }^{1}$ Department of Surgery, Medical School of University of Pécs, Hungary, Ifjúság útja 13, 7624 Pécs, Hungary

${ }_{2}$ Monklands District General Hospital, Monkscourt Avenue, Airdrie, Lanarkshire, ML6 0JS, Scotland

Received: 21 March 2002/Accepted in final form: 1 April 2002/Online publication: 27 June 2002

DOI: $10.1007 / \mathrm{s} 00464-002-4218-1$

Abstract. Colorectal manifestation of von Recklinghausen's disease is a rare entity. The typical dumbbell shape of the lesion has been described previously, but only from a resected cecal specimen. The authors present the endosonographic appearance of a rectal neurofibroma, which shows a similar hourglass shape, thus facilitating the diagnosis.

Key words: Neurofibroma - von Recklinghausen's disease - Rectal ultrasound

Correspondence to: M. Kassai

\section{What's right when the gallbladder's left?}

\section{A case report}

D. Gui, S. Magalini, F. Prete, D. Sermoneta

Department of Surgery, Catholic University, Policlinico Gemelli, Largo Agostino Gemelli 8, 00168, Rome, Italy

Received: 21 March 2002/Accepted in final form: 1 April 2002/Online publication: 27 June 2002

DOI: $10.1007 / \mathrm{s} 00464-002-4217-2$

Abstract. During laparoscopic cholecystectomy for cholelithiasis in a 40-year-old woman, we came upon a case of incidentally discovered left-sided gallbladder (LSG). Two anatomic variants of LSG are known: (a) "true LSG," in which, according to Gross, an accessory gallbladder originates from the left hepatic duct (LHD), the right embryonic bud is readsorbed, and the cystic duct joins either the CBD from the left or the LHD directly. Otherwise, a normal right-sided gallbladder adheres to the inferior left hepatic lobe, and the cystic duct joins the CBD from the right side (as in our case); (b) gallbladder under the fourth hepatic segment, medial to a right-sided round ligament, probably resulting from a prenatal obliteration of the right umbilical vein. Left-sided gallbladder is a paraphysiologic condition that when identified before surgery, must be studied by CT or MRI, when incidentally discovered during surgery must be promptly recognized by the surgeon, who must be aware of the unpredictable confluence of the cystic duct into the CBD. The following operative precautions are useful for avoiding a lesion of the CBD: The surgeon should start dissection of Calot's triangle as close as possible to the gallbladder margin, prepare and clip the cystic duct as close as possible to the infundibulum, and a $30^{\circ}$ angled telescope. If in doubt, the surgeon should perform an intraoperative cholangiography.

Key words: Left-sided gallbladder - Laparoscopic cholecystectomy - Etiopathology of left-side gallbladder Correspondence to: D. Gui

\section{Gallstone ileus as a complication of acute cholecystitis}

\section{Laparoscopic diagnosis and treatment}

\section{F. Agresta, N. Bedin}

Department of General Surgery, Ospedale Civile, Vittorio Veneto (TV), Italy

Online publication: 27 June 2002

DOI: $10.1007 / \mathrm{s} 00464-002-4215-4$

Abstract. Gallstone ileus accounts for $1-4 \%$ of all cases of intestinal obstruction, with its incidence rising with age of patients. There is often a long delay between onset of symptoms (usually abdominal pain, vomiting, and bowel distension) and proper treatment, with a simple enterolithotomy as the one of choice. We report a case of an atypical gallstone ileus presented as a complication of acute cholecystitis, treated with a laparoscopic guided enterolithotomy. A 67-year-old woman on the 5th p.o. day after a laparoscopic procedure for an empyematous cholecystitis (no sign of fistula or duodenal perforation and a "negative" intraoperative cholangiography) presented continuous vomiting as the only symptoms of a subileus (radiographic diagnostic images negative for intestinal obstruction or intraluminal gallstone or duodenal fistula). A laparoscopic diagnostic approach revealed a gallstone in the distal jejunum. Through a $5 \mathrm{~cm}$ midline incision the intestine, including the gallstone, was brought out extracorporally and the stone was removed by a simple enterolithotomy. The postoperative course was uneventful and the patient had no complaint at a 1-year follow-up. We consider the laparoscopic approach, in patients with "abdominal emergencies," feasible and safe in experienced hands. It provides diagnostic accuracy as well as therapeutic capabilities, as in the case of gallstone ileus we have reported.

Key words: Gallstone ileus - Obstruction - Intestinal - Laparoscopy

Correspondence to: F. Agresta

\section{Hand-assisted laparoscopic anatomical left lobectomy using hemihepatic vascular control technique}

T. Kurokawa, ${ }^{1}$ H. Inagaki, ${ }^{2}$ J. Sakamoto, ${ }^{2}$ T. Nonami ${ }^{1}$

${ }^{1}$ Department of Surgery, Aichi Medical University, Yazako Karimata

21, Nagakute-cho, Aichi-gun, Aichi, Japan

${ }^{2}$ Department of Surgery, Aichi Prefecture Hospital, Kuriyado 18,

Kakemachi, Okazaki, Aichi, Japan

Received: 14 February 2002/Accepted in final form: 4 March 2002

Online publication: 27 June 2002

DOI: $10.1007 / \mathrm{s} 00464-002-4212-7$ 